\section{Cureus}

Received 07/05/2017

Review began 08/23/2017

Review ended 08/24/2017

Published 08/27/2017

\section{C) Copyright 2017}

Mashriqi et al. This is an open access article distributed under the terms of the Creative Commons Attribution License CC-BY 3.0., which permits unrestricted use, distribution, and reproduction in any medium, provided the original author and source are credited.

\title{
Xiphoid Process Variations: A Review with an Extremely Unusual Case Report
}

\author{
Faizullah Mashriqi ${ }^{1}$, Anthony V. D'Antoni ${ }^{2}$, R. Shane Tubbs ${ }^{3}$ \\ 1. Department of Molecular, Cellular, and Biomedical Sciences, CUNY School of Medicine 2. Department \\ of Molecular, Cellular and Biomedical Sciences, CUNY School of Medicine 3. Neurosurgery, Seattle \\ Science Foundation
}

$\square$ Corresponding author: Faizullah Mashriqi, fmashri000@citymail.cuny.edu Disclosures can be found in Additional Information at the end of the article

\section{Abstract}

The xiphoid process is a small bony feature of the anterior thoracic wall just inferior to the sternum corpus. Although the xiphoid process is commonly represented as a straight, fully ossified bone in educational textbooks, reports of anomalous processes flood the literature. The xiphoid process can be broad, thin, monofid, bifid, trifid, curved, or deflected and contain foramina. Variations can be mistaken for epigastric masses. Herein, we report an extremely unusual bifid xiphoid process that is deflected anteriorly. This case is discussed in the context of the misdiagnosis of xiphoid process variations and its importance to the clinician.

Categories: Radiology, Miscellaneous, Orthopedics

Keywords: xiphoid process, sternum, epigastric mass, bifid, variation

\section{Introduction}

The xiphoid process (also known as the xiphisternum) is located in the epigastrium region of the anterior thoracic wall. The xiphoid process articulates with the superiorly located sternum corpus at the xiphisternal joint. During the first half of life, this joint is categorized as a symphysis but eventually becomes a synostosis at $\sim 40$ years [1]. Anteriorly, the xiphoid process serves as the attachment point for fibers of the rectus abdominis muscle and the aponeurosis of the internal and external oblique muscles of the anterior abdominal wall [2]. The xiphoid process attaches to the linea alba inferiorly and the diaphragmatic slips, transversus thoracis, and costoxiphoid ligaments posteriorly [1-2]. At birth, the xiphoid process is a cartilaginous structure and ossification begins at around three years of life from the superior-most portion [2]. The perforating branches of the internal thoracic artery perfuse the entire sternum.

Understanding the variants of regional bones during invasive procedures or during the interpretation of radiological images is necessary for good clinical care. Anteriorly deflected xiphoid processes can be mistaken for epigastric masses. Moreover, there have been reports of cardiac tamponade in patients with xiphoid foramina following acupuncture procedures that pierced the pericardial sac [1]. We present here a very unusual cadaveric case with an anteriorly deflected xiphoid process and review the literature regarding other malformations of this bony protuberance.

\section{Case Presentation}

During a routine dissection of the abdominothoracic region in an adult male cadaver, aged 78 years at death, an unusual protuberance in the epigastrium was observed. The cause of death of 


\section{Cureus}

the specimen was kidney failure with a history of diabetes and peripheral vascular disease. No signs of trauma, either recent or old, were identified in the area of the protuberance. Additionally, no signs of past surgical intervention to the region were observed. With continued deeper dissection, a $3 \mathrm{~cm}$ bony prominence was exposed. This was found to be continuous and an anterior extension of the xiphoid process (Figure 1). The linea alba was seen to be tented more superficially and was attached at the tip of the abnormally shaped xiphoid process. No other pathology of the abdominal region was noted other than a small left inguinal hernia, which was easily reduced.

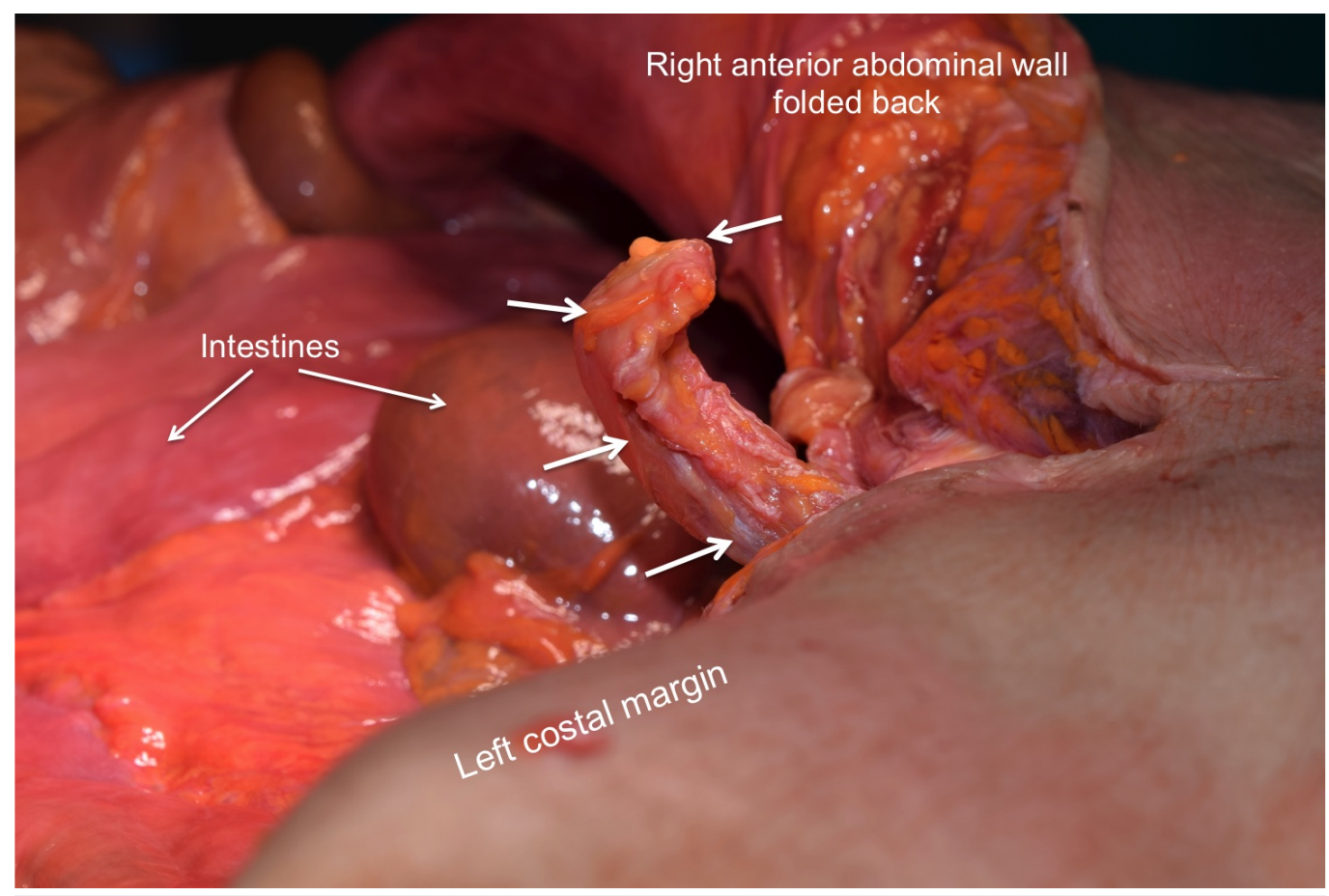

\section{FIGURE 1: Superolateral view of the xiphoid process identified during routine anatomical dissection}

No signs of previous trauma or surgery were identified around the xiphoid process. Note the acute angulation of the xiphoid process with a protuberance anteriorly. Arrows outline the congenitally deformed xiphoid process.

\section{Discussion}

The etymology derives from the Greek word "xiphos" meaning straight sword [1], but this is a misnomer, as the xiphoid process is the most variable sternal element and regularly takes on many forms. The xiphoid process can be broad, thin, monofid, bifid, trifid, curved, deflected, and contain foramina [1-3]. Current textbooks and human anatomy atlases depict the xiphoid process as a single, straight, fully ossified bone on the inferior aspect of the sternum corpus. In actuality, significant variation is reported in the literature [1-4].

The variation in xiphoid morphology was demonstrated using multidetector computed tomography (MDCT) in a 500 patients sample [2]. This study found that only $33.2 \%$ of patients had a xiphoid process that was in the same plane as the sternum corpus. Ventrally deflected processes were present in $65.4 \%$ of the patients. Monofid, bifid, and trifid processes were found in $62.6 \%, 32.8 \%$, and $4.6 \%$ of the patients, respectively. Approximately three percent of xiphoid processes were found to curve at the end, either dorsally or ventrally, and resembled a hook. A 
unique finding of this study was a reverse "S" shaped xiphoid process, which was found in $<1 \%$ of patients [2]. The prevalence of these different xiphoid process types is more or less consistent in the literature [3].

Reports of xiphoid foramina flood the literature [1-4]. Xiphoid foramina were present in 43.2\% of the patients in one study [2] and in $27.4 \%$ of patients in a second study [3]. Most subjects had one xiphoidal foramen, less than seven percent of the subjects had two xiphoidal foramina, and less than two percent of the subjects had three xiphoidal foramina [2]. An interesting case report from India showed a large $(1.6 \mathrm{~cm}$ by $1.4 \mathrm{~cm})$ foramen on a non-ossified xiphoid process that was pear shaped [1]. Pseudoforamina are another common reporting in the literature for the xiphoid process. A pseudoforamen represents the incomplete fusion of the xiphoid process to the sternum corpus. One study reported $3.6 \%$ of patients with a pseudoforamen [3] and a second study reported $1.2 \%$ of patients with the same anomaly [2]. Finally, the calcification of the costoxiphoid ligaments occurs in $\sim 10 \%$ of patients and was mostly identified in patients over 50 years of age [3].

\section{Conclusions}

Clearly, anomalies in the xiphoid process are common, and significant interindividual variation should be expected. The close proximity of the xiphoid process to thoracic structures and abdominal structures requires a thorough understanding of these easily misdiagnosed variations. We reported here a highly unusual bifid xiphoid process with an anterior deflection, which presented as an unusual epigastric protuberance prior to dissection.

\section{Additional Information \\ Disclosures}

Human subjects: Consent was obtained by all participants in this study. Conflicts of interest: In compliance with the ICMJE uniform disclosure form, all authors declare the following:

Payment/services info: All authors have declared that no financial support was received from any organization for the submitted work. Financial relationships: All authors have declared that they have no financial relationships at present or within the previous three years with any organizations that might have an interest in the submitted work. Other relationships: All authors have declared that there are no other relationships or activities that could appear to have influenced the submitted work.

\section{References}

1. Kumar NS, Bravian D, More AB: Xiphoid foramen and its clinical implication. Int $\mathrm{J}$ Anat Res. 2014, 2:340-343. https://www.ijmhr.org/ijar_articles_vol2_2/IJAR-2014-431.pdf.

2. Akin K, Kosehan D, Topcu A, Koktener A: Anatomic evaluation of the xiphoid process with 64-row multidetector computed tomography. Skeletal Radiol. 2011, 40:447-452. 10.1007/s00256-010-1022-1

3. Yekeler E, Tunaci M, Tunaci A, Dursun M, Acunas G: Frequency of sternal variations and anomalies evaluated by MDCT. AJR Am J Roentgenol. 2006, 186:956-960. Accessed: June 28, 2017: 10.2214/AJR.04.1779

4. Bermio VS, Hemalatha JGA: Congenital foramen in the body of the sternum . Int J Anat Res. 2014, 2:545-548. https://www.ijmhr.org/ijar_articles_vol2_3/IJAR-2014-483.pdf. 